\title{
IMPROVED REAL-TIME FACE RECOGNITION BASED ON THREE LEVEL WAVELET DECOMPOSITION-PRINCIPAL COMPONENT ANALYSIS AND MAHALANOBIS DISTANCE
}

\author{
${ }^{1}$ Edy Winarno, ${ }^{1}$ Agus Harjoko, ${ }^{2}$ Aniati Murni Arymurthy and ${ }^{1}$ Edi Winarko \\ ${ }^{1}$ Department of Computer Science and Electronics, Gadjah Mada University, Yogyakarta, Indonesia \\ ${ }^{2}$ Faculty of Computer Science, Universitas Indonesia, Depok, Indonesia
}

Received 2013-12-03; Revised 2014-01-07; Accepted 2014-01-17

\begin{abstract}
The development of research in the field of real-time face recognition is a study that is being developed in the last decade. Face recognition is used to identify person from an image or video. Recognition rate and computation time of real-time face recognition is one of the big challenges that must be developed. This study proposes a model of face recognition using the method of feature extraction by combining three level wavelet decomposition and Principal Component Analysis (PCA) and using the method of mahalanobis distance for the classification section (3WPCA-MD). A 3-level wavelet decomposition is used to decompose images by reducing the resolution used for those images. Using wavelet decomposition up to level 3 will produce an image with a very low resolution so as to reduce the value of the resulting computation time to be processed using PCA. Mahalanobis distance method is used to determine the degree of similarity among the features to produce a more optimal face recognition. Based on the results of experiments that have been done, they generated improved face recognition with high face recognition accuracy of up to $96 \%$ in average and produced faster computation results of face recognition if compared to ordinary PCA method. The average computation speed value obtained using the method of 3WPCA-MD was 5-7 milli-second (ms) for each face recognition process.
\end{abstract}

Keywords: Face Recognition, Real-Time, Wavelet, PCA, Mahalanobis

\section{INTRODUCTION}

Facerecognition system is an application of computer technology to make detection and recognition of detected human faces. Face recognition system is a way to recognize human faces of both still images and moving images such as real-time videos taken from a camera or webcam. Usually, face detection uses algorithm that is able to classify human faces and recognize the identity of the detected faces.

The method of face recognition system has been carried out in previous studies initiated by the face recognition system using eigenfaces as conducted by Turk and Pentland (1991). Research on face recognition for the benefit of humans has been done mainly for various purposes such as security system, surveillance, general identity verification, criminal justice systems, image database investigations, "smart card" applications, multi-media environments, video indexing and witness face reconstruction (Jafri and Arabnia, 2009).

According to Jafri and Arabnia (2009), based on the methodology of data acquisition, face recognition is divided into 3 categories: Face recognition method based on image intensity; face recognition method that works based on video sequences and face recognition method that requires other sensors such as 3D information and infrared imagery.

In the technique of face recognition, there are three main stages of the process to produce a system that can recognize human faces, namely, preprocessing, feature extraction and classification. The big challenges that must be developed in face recognition field are recognition rate and computation time of real-time face recognition. Corresponding Author: Edy Winarno, Department of Computer Science and Electronics, Gadjah Mada University, Yogyakarta, Indonesia 


\section{RELATED WORKS}

Some of related work that has been done by researcher in face recognition especially in feature extraction field can be found in previous research that mentioned below. Wei et al. (2009) developed a model of facial recognition using a combination of wavelet and PCA for the feature extraction, while for the classification, they used Support Vector Machines (SVM). Based on the results of the experiments conducted, face recognition accuracy of up to $92 \%$ was obtained. Chan et al. (2010) developed a framework of facial biometric use Principal Component Analysis (PCA) and Linear Discriminant Analysis (LDA). This research result an accuracy of face recognition using PCA achieve $91.9 \%$.

Sakthivel and Rajaram (2011) applied wavelet decomposition technique to enhance input images for reducing loss of classification performance. The research can produce accuracy of face recognition $91.87 \%$ in average. Chitaliya and Trivedi (2010) developed a model of face recognition and object classification using wavelet-PCA as feature extraction and using Euclidean distance and neural networks distance for the classification. This study used 1-level Discrete Wavelet Transform (DWT) for the original image used for its feature extraction. The results of this study generated facial recognition accuracy of up to $93.3 \%$.

In classification field, Ji et al. (2009) developed a model of face recognition based on sparse representation using Mahalanobis distance as the classification. The research can prove that the method of Mahalanobis distance is a classification method for better face recognition compared to the Euclidean distance method and other classification methods such as neural networks.

Kapoor and Bhatia (2010) used correlation and Mahalanobis Distance as classification to develop the facial gesture recognition. The research produced gesture recognition capable of recognizing gestures in various expressions well. Mahalanobis distance was used as the classification capable of producing better recognition results compared to other classification methods such as City Block Distance (CBD), Euclidean Distance (ED) and Weighted Euclidean Distance (WED).

\section{PROPOSED METHOD}

From some of the research that had been done previously, no research had combined the methods of feature extraction and classification performed in realtime. Therefore this study tries to merge and combine some of those methods and implement the overall methods into a new model of improved face recognition.
This study proposes a model of face recognition using feature extraction method by combining three level wavelet decomposition and Principal Component Analysis, where for the classification stage Mahalanobis distance is used (3WPCA-MD). There are four steps to perform the process of 3WPCA-MD namely: (1) conducting the process of facial image preprocessing, (2) performing the dimensional reduction process of the image with 3 level wavelet decomposition, (3) carrying out the process of feature extraction using PCA, (4) doing the classification using Mahalanobis distance. The proposed face recognition model in this study is shown in Fig. 1.

\subsection{Preprocessing}

In this study, we use Haar cascade classifier method to localize the face area (Padilla et al., 2012). In the preprocessing stage, we combine four steps:

- Cropping the facial image in the rectangle area of the face detection process

- Changing the facial image from the Red Green Blue (RGB) mode to grayscale to produce a grayscale image from a color image

- Resizing the facial image with the size of $160 \times 160$ pixels

- Performing image alignment and normalization of the brightness and contrast level using histogram equalization process

The preprocessing stage that conducted in this study is shown in Fig. 2.

\subsection{Feature Extraction}

The feature extraction method combines the methods of three level wavelet decomposition and Principal Component Analysis (PCA). We expect that this combining method will result a better model of face recognition than using PCA only. The combining method will result a smaller dimensional reduction of facial image resolution at $20 \times 20$ pixels. The process of combining the method of 3-level wavelet decomposition and PCA is shown in Fig. 3.

Using three level wavelet decomposition will produces an image with low resolution and will reduces the computation time before processed using PCA. The advantage of the use of PCA is to produce a better face recognition which has been tested in previous studies. However, if using only PCA will generate computation values that are too heavy since it uses very large matrix. 
Edy Winarno et al. / Journal of Computer Science 10 (5): 844-851, 2014

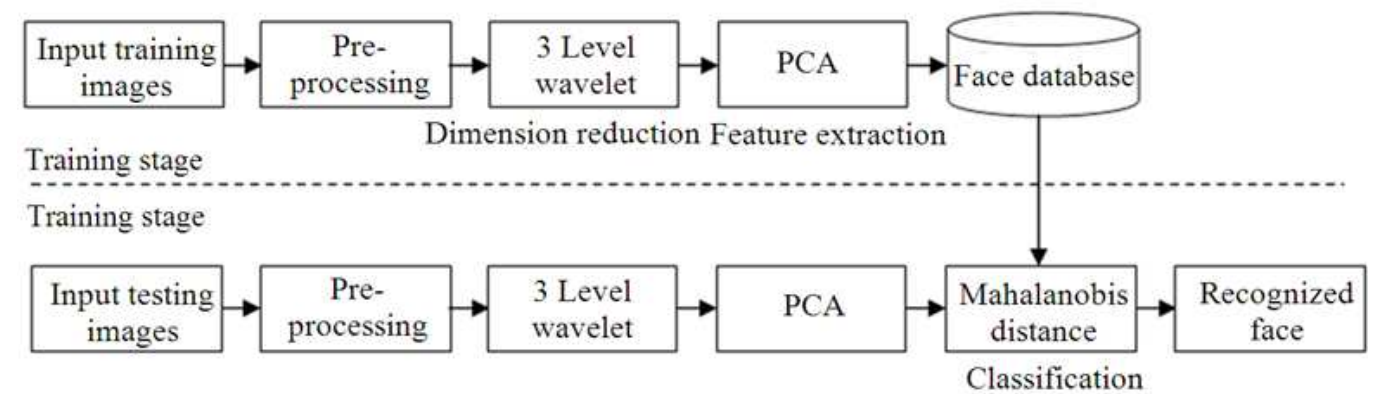

Fig. 1. 3WPCA-MD of face recognition



Fig. 2. Preprocessing

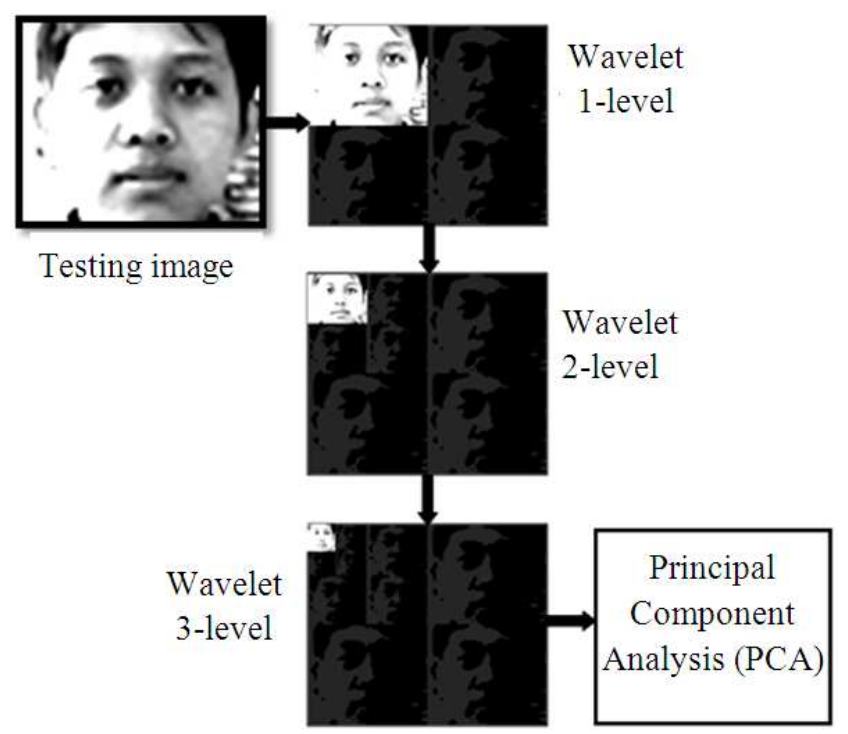

Fig. 3. Three level wavelet decomposition process 


\subsubsection{Three Level Wavelet Decomposition}

The method of wavelet that used in this research is Haar wavelet (Daubechies-1). We use 3-level of Haar wavelet decomposition to increase the speed of computation time to be processed using PCA. Wavelet is derived from a scaling function that can be composed from multiple copies of itself where dilations, translations and scaling have been performed. The dilation equation from this function is considered as the basis for the theory of wavelet. The dilation equation can be seen in Equation (1):

$\Phi(\mathrm{x})=\sum \mathrm{C}_{\mathrm{K}} \Phi(2 \mathrm{x}-\mathrm{k})$

From the Equation1, the wavelet equation can be formulated as Equation (2):

$\Phi^{0}(\mathrm{x})=\sum_{\mathrm{k}}(-1)^{\mathrm{k}} \mathrm{C}_{1-\mathrm{k}} \Phi(2 \mathrm{x}-\mathrm{k})$

Wavelet decomposition process can be described using the orthonormal bases of $\mathrm{v}_{0}, \mathrm{v}_{1}, \mathrm{v}_{2}$ and can be provided as matrix of $\mathrm{v}_{0}, \mathrm{v}_{1}, \mathrm{v}_{2}$ and $\mathrm{v}_{3}$. If there is a matrix:

$x=\left[\begin{array}{l}a \\ b \\ c \\ d\end{array}\right]$

Then the above matrix can be represented in Equation (3):

$\mathrm{x}=\mathrm{av}_{0}+\mathrm{bv}_{1}+\mathrm{cv}_{2}+\mathrm{dv_{3 }}$

To represent a matrix as a linear combination of matrix-vector in the wavelet, the value of $a, b, c$ and $d$ can be determined using Equation (4):

$\left[\begin{array}{l}\mathrm{x}_{0} \\ \mathrm{x}_{1} \\ \mathrm{x}_{2} \\ \mathrm{x}_{3}\end{array}\right]=\mathrm{a}\left[\begin{array}{l}1 \\ 1 \\ 1 \\ 1\end{array}\right]+\mathrm{b}\left[\begin{array}{l}1 \\ 1 \\ -1 \\ -1\end{array}\right]+\mathrm{c}\left[\begin{array}{l}1 \\ -1 \\ 0 \\ 0\end{array}\right]+\mathrm{d}\left[\begin{array}{l}0 \\ 0 \\ 1 \\ -1\end{array}\right]$

Then, the following equations can be derived from the following Equation (4):

$$
\begin{aligned}
& \mathrm{x}_{0}=\mathrm{a}+\mathrm{b}+\mathrm{c} \\
& \mathrm{x}_{1}=\mathrm{a}+\mathrm{b}+\mathrm{c} \\
& \mathrm{x}_{2}=\mathrm{a}-\mathrm{b}+\mathrm{d} \\
& \mathrm{x}_{3}=\mathrm{a}-\mathrm{b}-\mathrm{d}
\end{aligned}
$$

Which generated:

$$
\begin{aligned}
& \mathrm{x}_{2}-\mathrm{x}_{3}=2 \mathrm{~d} \\
& \mathrm{x}_{0}-\mathrm{x}_{1}=2 \mathrm{x} \\
& \left(\mathrm{x}_{0}+\mathrm{x}_{1}\right)-\left(\mathrm{x}_{2}+\mathrm{x}_{3}\right)=4 \mathrm{~b} \\
& \left(\mathrm{x}_{0}+\mathrm{x}_{1}\right)+\left(\mathrm{x}_{2}+\mathrm{x}_{3}\right)=4 \mathrm{~b}
\end{aligned}
$$

Hence, it can be concluded that:

$$
\begin{aligned}
& d=1 / 2\left(x_{2}-x_{3}\right) c=1 / 2\left(x_{0}-x_{1}\right) \\
& b=1 / 2\left(1 / 2\left(x_{0}+x_{1}\right)-1 / 2\left(x_{2}+x_{3}\right)\right) \\
& a=1 / 2\left(1 / 2\left(x_{0}+x_{1}\right)+1 / 2\left(x_{2}+x_{3}\right)\right)
\end{aligned}
$$

The transformation process of decomposition and reconstruction that have been done on a vector, then is followed by transformation on the image. An image can be considered as a two-dimensional matrix. The transformation process then is conducted on the lines and the columns in the image, as shown in Fig. 4.

LL is the result of lowpass towards rows and columns, LH is the result of lowpass towards lines followed by highpass towards column. HL is the result of highpass towards rows followed by lowpass towards columns, whereas $\mathrm{HH}$ is the result of highpass towards rows and columns.

Decomposition can be done to three levels $\left(a^{0}\right.$ until $\left.a^{2}\right)$. The wavelet decomposition process is shown in Fig. 5.

LL1, HL1, LH1 and HH1 are the output of the decomposition level-1, LL2, HL2, LH2 and HH2 are the output of decomposition level-2, LL2 can be decomposed using level-3 into LL3, HL3, LH3 and HH3.

LL is called Approximation part (A), LH is Vertical details (V), HL is Horizontal details $(\mathrm{H})$ and $\mathrm{HH}$ is Diagonal details (D).

\subsubsection{Principal Component Analysis (PCA)}

PCA is a feature extraction model that serves to reduce features of an image before the classification process is carried out. PCA method can be briefly described as follows (Nixon and Aguado, 2012):

- Find the characteristics matrix of data $c_{x}$. Each matrix column defines feature vectors

- Calculate the covariance matrix $\sum_{\mathrm{x}}$. This matrix provides information about linear independence among features

- Find the eigenvalues using the equation of characteristic $\operatorname{det}\left(\lambda_{\mathrm{i}} \mathrm{I}-\sum_{\mathrm{x}}\right)=0$. This value forms a diagonal covariance matrix $\sum_{\mathrm{y}}$. Each element in the matrix is the variance of the transformed data 




Fig. 4. Transformation of rows and columns in an image

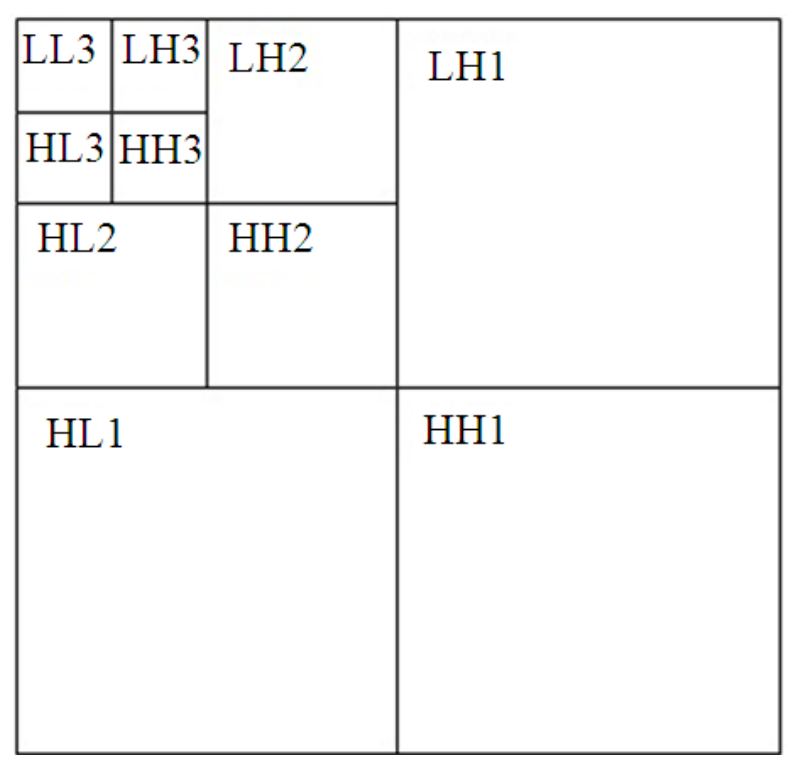

Fig. 5. Decomposition using wavelet

- Find eigenvectors using $\mathrm{w}_{\mathrm{i}}$ in $\left(\lambda_{\mathrm{i}} \mathrm{I} \sum_{\mathrm{x}}\right) \mathrm{w}_{\mathrm{i}}=0$ every eigenvalue. Make normalization and linearly independent for eigenvectors. Transformation of $\mathrm{W}$ is produced by calculating columns of the eigenvectors

- Find transformation characteristics by counting $c_{y}=c_{x}$ $\mathrm{W}^{\mathrm{T}}$

- Select a high value character of $\lambda_{i}$. Since $\lambda_{i}$ measures the value of the variance and thus characteristics with high values of $\lambda_{i}$ will also have high variance

\subsection{Classification}

In the classification stage, we use the Mahalanobis distance in order to generate the more accurate value of face recognition and having a faster computing time than extraction PCA only. Mahalanobis distance will be used to determine the degree of similarity among the features to produce more optimal face recognition. We expect that this study will result a model of reliable face recognition that eventually will affect the accuracy of face recognition. Several previous studies show that the Mahalanobis distance is better than some other classifications (Kapoor and Bhatia, 2010). Mahalanobis distance can also be used as a reliable classifier especially to anticipate variations in illumination and differences in facial expression of face.

If $\mathrm{D}^{2}$ is MD (Mahalanobis Distance), $\mathrm{m}$ is vector of mean, $\mathrm{x}$ is data of vector, $\mathrm{T}$ is vector transpose, $\mathrm{C}^{-1}$ is inverse covariance matrix and then Mahalanobis distances can be calculated as shown in Equation (5):

$$
D^{2}=(x-m)^{T} C^{-1}(x-m)
$$

\section{EXPERIMENTAL RESULTS}

The proposed 3WPCA-MD is implemented using 40 faces in database storage. Each of face has 5 positions in different angles. We use Intel core i5 2,6Ghz to process this experiments. The testing results of this experiment are (1) a comparison of recognition rates based on classification method, the use of image resolution size and the use of features, (2) a comparison of computation time based on different image resolution size and classification method.

At the first, we implement preprocessing stage to crop the facial image in the rectangular area of the face detection process, then the image mode was changed from Red Green Blue (RGB) to gray-scale. The facial image resizing process was performed with a size of $160 \times 160$ pixels, then the histogram equalization process was conducted to normalize the image's brightness and contrast level.

At the second, we combine the method of three level wavelet decomposition and Principal Component Analysis (3WPCA). This method is used to generate the feature extraction results from the previously processed facial image in the preprocessing stage. Source images and images that resulted from the wavelet level 1,2 and 3 is shown in Fig. 6.

At the last step, we use Mahalanobis Distance as classification to compare database of faces and testing data. 


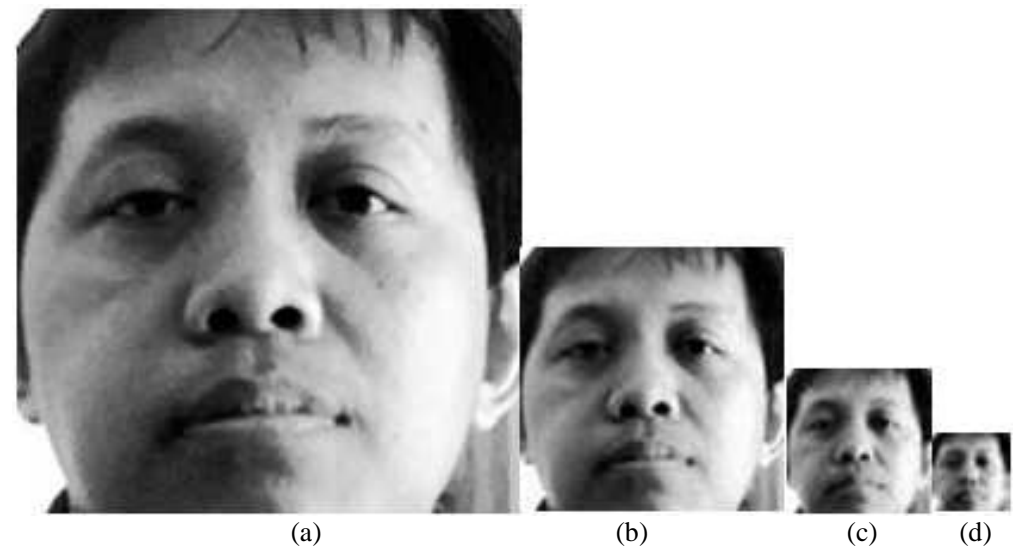

Fig. 6. (a) Source images $(160 \times 160$ pixels), (b) Wavelet level $1(80 \times 80$ pixels), (c) Wavelet level $2(40 \times 40$ pixels), (d) Wavelet level $3(20 \times \times 20$ pixels $)$

Table 1. Recognition rates using several input images based on feature extraction and classification

\begin{tabular}{lllll}
\hline Input image resolution & PCA-Euclidean & PCA-Mahalanobis & 3WPCA-Euclidean & 3WPCA-Mahalanobis \\
\hline $320 \times 320$ & 93.7 & 94.2 & 94.7 & 96.5 \\
$200 \times 200$ & 93.1 & 93.5 & 94.6 & 96.2 \\
$160 \times 160$ & 91.8 & 92.4 & 93.8 & 95.7 \\
\hline
\end{tabular}

\subsection{Comparison of Recognition Rate}

In the comparison of recognition rate, we use several input image resolution (1) $320 \times 320$ pixels, (2) $200 \times 200$ pixels and (3) $160 \times 160$ pixels. All of input image resolution are used to examine the comparison between the proposed method (3WPCA) and PCA method. We also compare the comparison of recognition rate using Euclidean Distance and Mahalanobis Distance as classification.

The performance results of face recognition can be seen in Table 1. The testing results can be concluded that the use of 3WPCA method is better than PCA method and the use of Mahalanobis distance on 3WPCA is better than Euclidean Distance. It shows that the combining of three level Wavelet-PCA and Mahalanobis Distance (3WPCA-MD) can result the better of face recognition rate about $95.7 \%$.

\subsection{Comparison of Computation Time}

In the comparison of computation time, we use several input image resolution (1) $320 \times 320$ pixels and (2) $160 \times 160$ pixels. Then, we use four types of wavelet filters: (1) normal, (2) wavelet level 1, (3) wavelet level 2 and (4) wavelet level 3. We use Mahalanobis distance and Euclidean Distance as classification to compare the testing computation time. The testing computation time is conducted to determine the time that needed to recognize one's face.

The results of testing computation time by comparing the classification of Euclidean distance and Mahalanobis Distance using $320 \times 320$ pixels of face image can be seen in Fig. 7. It shows that the use of Mahalanobis distance in wavelet level 1-3 will produce computation time better than Euclidean distance. The use of Mahalanobis distance on wavelet level 3 can result computation time about $8.501 \mathrm{~ms}$, it is better than the use of Euclidean distance that can result $9.748 \mathrm{~ms}$ of computation time.

The results of the testing computation time using $160 \times 160$ pixels of face image is shown in Fig. 8. We can see that the testing computation time results for the wavelet level 3 is better than the measurement of the testing computation time in the wavelet level 1 , level 2 and normal image sizes. The use of $160 \times 160$ pixels on Mahalanobis distance can result $5.56 \mathrm{~ms}$ of computation time and the use on Euclidean distance can result $6.24 \mathrm{~ms}$ of computation time.

From the testing of computation time in Fig. 7 and 8, we can conclude that the use of 3WPCAMahalanobis distance can produce faster computation speed than using 3WPCA-Euclidean distance and the use of $160 \times 160$ pixels will result a better computation time than using $320 \times 320$ pixels. 




Fig. 7. Comparison of computation time on WPCA based on classification using $320 \times 320$ pixels of face image

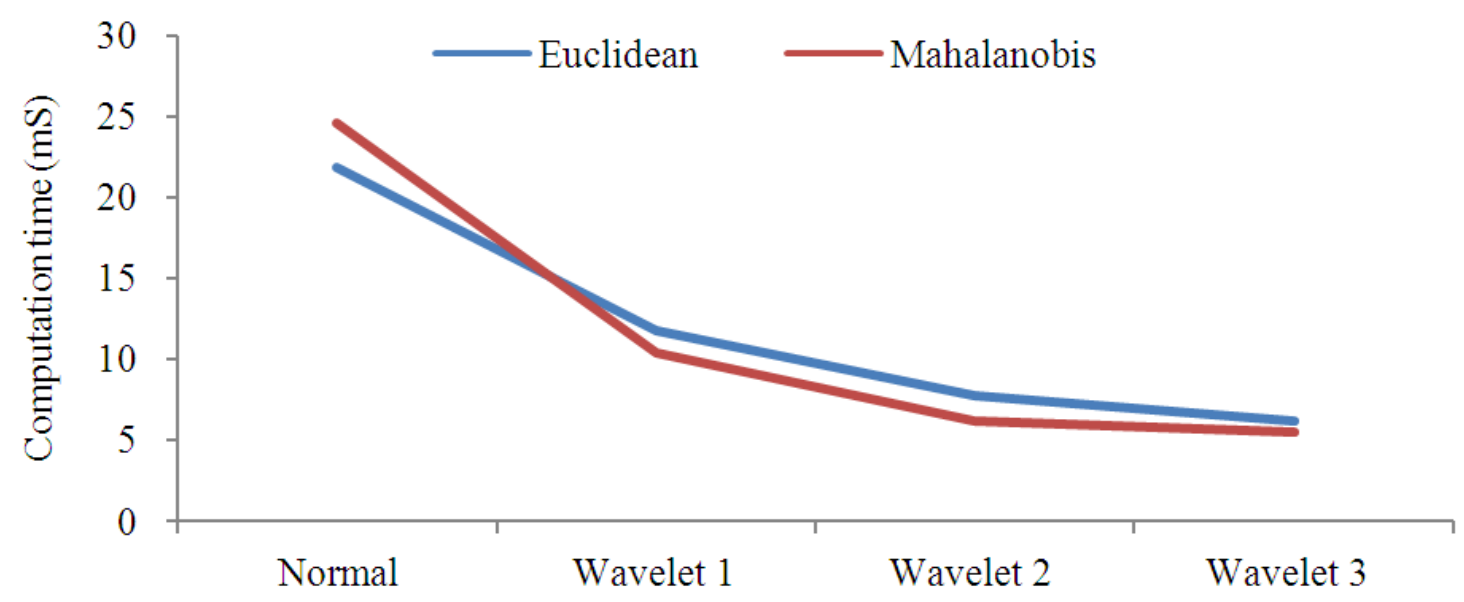

Fig. 8. Comparison of computation time on WPCA based on classification using $160 \times 160$ pixels of face image

\section{CONCLUSION}

The proposed 3WPCA-MD face recognition in this study can produce a good performance of face recognition. The 3WPCA-MD method also generated a faster testing computation time than other face recognition methods. The 3-level wavelet with the size of $20 \times 20$ pixels still gave good recognition result about $95.7 \%$ and good computation speed up to 5-7 milli-second (ms).

\section{REFERENCES}

Chitaliya, N.G. and A.I. Trivedi, 2010. An efficient method for face feature extraction and recognition based on contourlet transform and principal component analysis using neural network. Int. J. Comput. Applicat.
Chan, L.H., S.H. Salleh and C.M. Ting, 2010. Face biometrics based on principal component analysis and linear discriminant analysis. J. Comput. Sci., 6: 693-699. DOI: 10.3844/jcssp.2010.693.699

Jafri, R. and H. R. Arabnia, 2009. A survey of face recognition techniques. J. Inform. Process. Syst.

Ji, Y., T. Lin and H. Zha, 2009. Mahalanobis distance based non-negative sparse representation for face recognition. Proceedings of the International Conference on Machine Learning and Applications, Dec. 13-15, IEEE Xplore Press, Miami Beach, pp: 41-46. DOI: 10.1109/ICMLA.2009.50

Kapoor, S. and R. Bhatia, 2010. Facial gesture recognition using correlation and mahalanobis distance. Int. J. Comput. Sci. Inform. Security.

Nixon, M.S. and A.S. Aguado, 2012. Feature Extraction and Image Processing. 3rd Edn., Academic Press, Amsterdam, ISBN: 9780123978240, pp: 632. 
Padilla, R., C.F.F.C. Filho and M.G.F. Costa, 2012. Evaluation of Haar cascade classifiers designed for face detection. World Acad. Sci. Eng. Technol.

Sakthivel, S. and M. Rajaram, 2011. Improving the performance of machine learning based multi attribute face recognition algorithm using wavelet based image decomposition technique. J. Comput. Sci., 7: 366-373.

DOI: 10.3844/jcssp.2011.366.373
Turk, M. and A. Pentland, 1991. Eigenfaces for recognition. Int. J. Cognitive Neurosci., 3: 71-86.

Wei, L.X., Y. Sheng, W. Qi and L. Ming, 2009. Face recognition based on wavelet transform and PCA. Proceedings of the Pacific-Asia Conference on Knowledge Engineering and Software Engineering, Dec. 19-20, IEEE Xplore Press, Shenzhen, pp: 136138. DOI: 10.1109/KESE.2009.43 